\title{
La coopération Nord-Sud pour l'enseignement et la recherche : le cas des pays francophones du Sud-Est asiatique
}

\author{
Mokhtar Ben Henda, Université Bordeaux Montaigne
}

DOI : 10.51186/journals/ed.2020.10-2.e347

\section{Résumé}

Depuis 2015, un vaste projet de coopération francophone, sous couvert de l'Agence universitaire de la Francophonie (AUF), mobilise des acteurs/trices scientifiques de la recherche et de l'enseignement de pays européens et du sud-est asiatique. Des universités européennes francophones sont entrées conjointement avec des universités vietnamiennes, cambodgiennes et laotiennes dans une dynamique de collaboration active dans la perspective d'un transfert de technologies, mais aussi d'une mutualisation d'expériences dans la gouvernance universitaire par le numérique. Études, expertises, formations, publications et projets constituent des mécanismes d'amélioration de la qualité, de la pertinence et de l'efficience tant de la recherche que de l'enseignement pour les différents partenaires. Aujourd'hui, des équipes ad-hoc, des comités d'experts, des consortiums et des délégations internationales participent de la redéfinition d'une culture scientifique de la recherche et de l'innovation pédagogique par le numérique, faisant de la disparité des contextes et de l'asymétrie entre les acteurs du Nord et du Sud un vecteur d'innovation et d'enrichissement d'expériences.

Mots-clés : Francophonie, coopération Nord-Sud, Sud-est asiatique, transfert de technologies, gouvernance universitaire

\section{Abstract}

Since 2015, Agence universitaire de la Francophonie (AUF) has led an extensive cooperation project involving researchers and educators from French-speaking European and Southeast Asian countries. French-speaking universities in Europe, Vietnam, Cambodia and Laos have entered into a process of active collaboration with a view to transferring technologies and sharing experiences around university governance strategies and ICT optimization. Within this project, studies, expertise, training courses, publications and projects are being used to improve the quality, relevance and efficiency of both research and teaching for the concerned partners. Today, ad-hoc teams, committees of experts, consortia and international delegations are helping redefine a scientific culture of research and pedagogical innovation through digital technology, making the disparity of contexts and the asymmetry between the actors of the North and the South a driver for innovation and the enrichment of experiences. 
Keywords: Francophonie, North-South Cooperation, South-East Asia, technology transfer, university governance

\section{INTRODUCTION}

II est question dans cet article de faire un état des lieux et de discuter de la pertinence d'un certain nombre d'initiatives et de débats conceptuels et méthodologiques mis au cœur d'une série de projets pilotes auxquels nous avons contribués pendant les cinq dernières années dans le cadre d'une coopération entre des universités européennes francophones et des partenaires académiques du sud-est asiatique, membres de la Conférence régionale des recteurs des établissements membres de l'AUF en Asie-Pacifique (CONFRASIE). II est question en particulier de projets de gouvernance universitaire, notamment dans les domaines de l'analyse prospective, l'assurance qualité, la formation hybride et l'innovation pédagogique par les TIC. Ces projets qui ont marqué les initiatives francophones dans la région du Sud-est asiatique nous ont permis d'observer des formes d'influences socioculturelles particulières dans les pratiques de la recherche scientifique et de l'enseignement supérieur dans cette région du monde.

Nous nous concentrerons dans cet article sur trois des pays du Sud-Est asiatique, dits les pays CLV (Cambodge, Laos et Vietnam) ${ }^{1}$ par référence à une classification économique au sein même de l'ASEAN (Association des nations de l'Asie du Sud-Est) ${ }^{2}$. Ces trois pays ont fait l'objet d'une visite de terrain entre 2015 et 2016, et d'une étude exploratoire que nous avons réalisées au compte de l'Agence universitaire de la Francophonie pour étudier le taux d'introduction des TICE dans les pratiques éducatives universitaires de ces trois pays (Ben Henda, 2016b).

Pour apprécier pleinement les pressions et les opportunités auxquelles sont confrontés les établissements d'enseignement supérieur des pays CLV, il est aussi nécessaire de comprendre l'ensemble plus large des pressions qui influencent la croissance et le développement de l'enseignement supérieur et de la recherche dans la région, et dans le monde en général.

Sans prétendre analyser l'ensemble des problématiques auxquelles l'enseignement supérieur et la recherche font et devront faire face dans les pays CLV, nous nous proposons d'analyser, à l'aune de la situation actuelle, les défis d'une gouvernance universitaire générale qui vise des

\footnotetext{
${ }^{1}$ Les pays CLV font partie de la coopération CLMV (avec Myanmar en plus). II s'agit d'un cadre de coopération ouverte qui valorise les potentiels et les atouts de la coopération existant entre les pays CLMV, mais qui appelle aussi les autres pays membres de l'ASEAN à leur accorder une assistance dans le cadre d'accords bilatéraux de partenariats de développement. La $18^{\text {ème }}$ conférence des hauts responsables de l'Économie (SEOM) du Cambodge, du Laos, du Myanmar et du Vietnam (CLMV SEOM) a eu lieu le 12 janvier 2020 à Hanoï.

${ }^{2}$ Les 10 pays de l'ASEAN sont souvent classés en trois groupes : les pays à revenu élevé comme Singapour et Brunei ; l'ASEAN-4 pays de I'Indonésie, la Malaisie, les Philippines et la Thaillande ; et les pays CLMV du Cambodge, de la République démocratique populaire du Laos, du Myanmar et du Viet Nam.
} 
objectifs de qualité de haut niveau. La question de la qualité est en effet un fil conducteur des initiatives d'accompagnement programmé par la Francophonie dans la région.

\section{UN CONDENSÉ SUR L'ÉDUCATION ET L'ENSEIGNEMENT SUPÉRIEUR DANS LES PAYS DU SUD-EST ASIATIQUE}

Le cadre éducatif des pays CLV ne peut se soustraire à son contexte géopolitique naturel, celui de l'Asie pacifique, région qui foisonne de projets et de programmes de coopération avec des acteurs et des organismes d'Amérique, d'Europe et d'Australie. Le programme phare qui pèse de tout son poids sur les orientations stratégiques des trois pays et qui leur donne les grandes lignes d'une politique de développement économique et social est sans doute celui de I'ASEAN. Cette structure créée le 8 aout 1967 a pour but de renforcer les liens existants de solidarité et de coopération régionale en vue de faire avancer les intérêts généraux de la région qui couvrent des domaines multiples allant des questions politiques aux sujets économiques et culturelles, aux préoccupations environnementales et questions de sécurité jusqu'aux sujets du développement de l'éducation et des technologies numériques (Ben Henda, 2016a).

La politique générale de l'ASEAN s'est largement basée sur un programme de renforcement des technologies numériques à travers un « Plan Directeur ASEAN pour les TIC » (ASEAN ICT Master Plan ${ }^{3}$ ) dans ses deux phases successives : 2010-2015 et 2016-2020. Cependant, la situation de l'éducation et des technologies de l'information et de la communication (TIC) en Asie pacifique n'a pas été uniquement une affaire des pays de l'ASEAN. Plusieurs organismes internationaux de développement et d'aide au développement s'y sont également intéressés. Autant I'UNESCO que le PNUD ou la Banque Mondiale et l'OCDE, partant de leurs vocations internationales respectives, investissent lourdement dans les études stratégiques de cette région du monde en rapport avec des sujets sensibles de l'économie mondiale, de la fracture numérique ou des inégalités d'accès à l'éducation. Ils publient beaucoup d'études et de rapports mettant en avant l'état des lieux des télécommunications, des TIC et de l'éducation comme indicateurs clé de développement économique et social.

Dans un rapport de I'UNESCO, publié en 2014, il est souligné, compte tenu de la grande diversité des législations, des politiques et des systèmes de gestion de l'éducation dans la région de l'Asie pacifique, qu'il existe de grandes disparités dans la manière dont les pays de la région gèrent leurs systèmes éducatifs, particulièrement l'enseignement supérieur (UNESCO, 2014). Dans les trois pays CLV, les gouvernements sont de plus en plus confrontés à une forte demande sociale pour élargir l'accès à l'enseignement supérieur. Toujours est-il que plusieurs autres études expliquent que le soutien à l'enseignement supérieur avait connu une baisse entre 1990 et 2005 en raison du consensus international atteint lors de la «Conférence mondiale sur l'éducation pour tous » de 1990 selon lequel « le principal défi du

\footnotetext{
${ }^{3}$ Cf. ASEAN ICT Masterplan 2020, https://www.asean.org/storage/images/2015/November/ICT/15b\%20-\%20AIM\%202020_Publication_Final.pdf
} 
développement de cette époque était d'élargir l'accès à l'éducation de base ${ }^{4}$ » (Hirosato \& Kitamura, 2009, p. 92).

Depuis 2006, une nouvelle réforme de l'enseignement supérieur au Vietnam (HERA/Higher Education Reform Agenda), a proposé une nouvelle vision. Selon Cerbelle (2016) :

Le programme de réforme envisage un système qui, d'ici à 2020, aura triplé ou quadruplé sa taille actuelle, sera mieux géré et intégré, plus flexible dans son offre, plus équitable, financièrement plus autonome, davantage orienté vers la recherche, la commercialisation de sa recherche et de ses offres de formations, et plus conforme aux références internationales. (p. 245)

En 2013, le Ministère de l'Éducation et de la Formation (MEF) du Vietnam a également lancé le programme de bourses doctorales « 20000 docteurs en 2020 » en partenariat avec l'Ambassade de France. Ce programme de bourses doctorales, arrêté en 2017, " a permis d'accompagner l'augmentation du nombre d'enseignants-chercheurs titulaires d'un diplôme de doctorat passé de 14\% en 2013 à 23\% en 2017 » (MAE, 2018, p. 4).

Un changement de paradigme a donc été introduit dans le secteur de l'éducation et de la recherche sous l'impulsion d'une nouvelle logique mondiale de globalisation et de coopération internationale. Aujourd'hui, comme dans de nombreux pays du monde, la qualité de l'éducation est encore un chantier ouvert en quête de conformité avec les normes nationales et internationales. Le renforcement des systèmes de gestion, y compris un soutien ciblé au secteur de l'enseignement privé, un financement public équitable et durable, et une gouvernance de qualité des établissements d'enseignement supérieur et de professionnel-les qualifié-es, deviennent essentiels pour garantir l'équité et la qualité de l'éducation dans les pays CLV. Au Vietnam, la politique de socialisation de l'éducation a donné lieu à un nouveau type d'institution d'enseignement supérieur : les établissements dits "à capitaux étrangers » créés depuis 2000 sur la base du Décret n 06/2000/ND-CP66 qui promulgue une coopération en matière d'investissement avec l'étranger dans des domaines variés, principalement l'éducation, la formation et la recherche scientifique :

Cette ouverture avait pour objectif de soulager la pression de la demande d'un enseignement supérieur de grande qualité, à laquelle les établissements locaux n'arrivaient pas à répondre. Le décret a permis l'arrivée d'institutions étrangères avec un investissement à 100\% de leur part ou en coopération avec des institutions locales. (Nguyen Thi Yhanh, 2015, p. 16)

\footnotetext{
${ }^{4}$ L'argument principal était que l'enseignement supérieur était cher et que la plupart des avantages revenaient aux étudiant-es elles/eux-mêmes plutôt qu'à la société en général. En moyenne, les pays à faible revenu dépensent 34 fois plus pour un-e étudiant-e dans l'enseignement supérieur que pour un-e élève dans l'enseignement primaire et 14 fois plus que ce qu'ils dépensent pour un-e élève dans l'enseignement secondaire. ${ }^{5}$ Un rapport de 2014 du ministère de l'Éducation et de la Formation du Vietnam indique toutefois que " la proportion de doctorants dans l'ensemble du secteur de l'enseignement supérieur n'est que d'environ 22\%, en particulier la proportion de professeurs titulaires d'un doctorat dans les écoles pédagogiques est trop faible, environ $3,4 \%$ ».
} 
Il y a eu ainsi une conscience générale de la nécessité d'améliorer le système d'enseignement s'appuyant sur les progrès accomplis jusqu'alors dans le domaine de l'éducation de base. Les pays CLV se rendent progressivement compte du renforcement nécessaire d'autres niveaux d'enseignement pour avoir une population bien éduquée et qualifiée, capable de contribuer efficacement au développement socioéconomique. Les besoins en compétences sont prévus répondre non seulement aux objectifs présents, mais également aux besoins encore inexistants dans un contexte mondial en changement rapide. Toute réussite de la mise en œuvre des politiques et des réformes de l'éducation repose largement sur des partenariats avec un certain nombre de parties prenantes aussi bien les gouvernements et le secteur privé que la société civile et les organisations nationales et internationales.

\section{UN CONDENSÉ SUR L’ÉTAT DE LA RECHERCHE SCIENTIFIQUE DANS LES PAYS DU SUD-EST ASIATIQUE}

Nous choisissons de partir sur ce point à partir d'un récent rapport publié en 2019 par l'Institut de l'Information Scientifique (ISI) qui couvre, outre les pays CLV, des pays développés comme la Chine, le Japon et la Corée du Sud (Adam, et al., 2019). Plusieurs études et rapports couvrent cette région du monde dans ses différents aspects socioéconomiques, y compris l'éducation et la recherche (Carroué, 2015 ; Nguyen, et al., 2017 ; Vuong, 2019), mais nous avons préféré porter notre choix sur le rapport de 2019 de l'ISI au vu de sa forte corrélation avec notre propre étude réalisée en 2016 (Ben Henda, 2016a).

Le rapport souligne que cette région contient des niveaux très différents de développement de la recherche qui, en termes d'investissements, reste généralement inférieure à celle de l'Europe. Alors qu'à Singapour, par exemple, la collaboration scientifique internationale n'est qu'un élément dans la stratégie nationale du développement de la recherche scientifique, dans les trois pays CLV, la coopération internationale constitue l'essentiel des activités de recherche de haute performance. Comparés aux «Dragons asiatiques » (Corée du Sud, Hong-Kong, Taïwan, Singapour) et aux «tigres asiatiques » (Indonésie, Malaisie, Philippines, Thaillande) $\wedge^{\wedge}$, les indicateurs de recherche pour les pays CLV aux " petites " économies restent très dépendants de leur implication dans les collaborations internationales. Pourtant, leurs activités de recherche, selon le même rapport, ont plus que doublé depuis 2009. Sur un total régional annuel d'environ 12000 articles (articles et revues), la production scientifique représente désormais plus de $8 \%$ des publications mondiales chaque année. À titre de rappel, les économies de recherche de taille moyenne sont l'Indonésie, le Bangladesh, les Philippines et le Sri Lanka, avec 1000 à 5000 communications par an alors que le Cambodge, le Myanmar, le Laos et le Brunei sont les quatre pays comptant moins de 500 articles par an. En revanche, à lui seul, le Vietnam aurait multiplié par cinq son volume de publications indexées depuis 2009 (Adam, et al. , 2019, p. 5).

\footnotetext{
${ }^{6}$ Le Vietnam est désormais listé parmi les « Tigres asiatiques » avec 244948 milliards \$USD en 2018.
} 
Cependant, si ces données démontrent une courbe exponentielle du développement de la recherche dans la région, les disparités entre la population, l'écart de développement entre les régions, les modèles économiques de l'éducation, etc., continuent à entraver un potentiel non atteint de développement dans de nombreux secteurs connexes qui ont un impact direct sur la qualité de la recherche scientifique et de l'enseignement supérieur (David \& Amey, 2020). La poursuite du développement de la recherche interne nécessiterait par exemple une réitération de l'effort de recherche réalisé à l'extérieur d'autant que des scientifiques expatrié-es de ces pays ont apporté individuellement des contributions importantes à la recherche dans leurs pays d'accueil (Adam, et al., 2019). Cette réitération devrait normalement stimuler l'infrastructure de l'enseignement supérieur et de la recherche qui devrait conduire à une croissance soutenue des capacités nationales de connaissances, réduire la dépendance à l'égard des partenariats internationaux et soutenir l'innovation technologique et la compétitivité économique (Nguyen Tan, 2017). Pour l'instant, la coopération internationale est encore à pied d'œuvre pour atteindre cet objectif d'autonomisation qui constitue, en fin de compte, l'un des objectifs prioritaires des réformes universitaires dans les pays CLV.

\section{LES DÉTERMINANTS SOCIOÉCONOMIQUES ET CULTURELS}

Plusieurs déterminants impactent le contexte dans lequel évoluent les domaines de l'enseignement et de la recherche dans les pays CLV. Outre un monopole politique d'État sur les grandes orientations stratégiques des domaines publics et privés, plusieurs caractéristiques socioculturelles, juridiques et économiques font de ces domaines des canevas complexes à gérer.

Le déterminant économique est sans doute celui qui impacte le plus la situation de l'enseignement et de la recherche. Avec des PIB en $2019^{7}$ (en millions de dollars US) qui sont parmi les moins élevés de la région (Cambodge : 27089 ; Laos : 18173 ; Vietnam : 261 921), comparés à la Chine (14 342 902) ou à l'Indonésie (1 119 190), les métiers de l'enseignement et de la recherche n'en sont pas épargnés. Encore loin des standards internationaux en termes de rémunération salariale ${ }^{8}$ et de valorisation sociale, ces deux secteurs d'activité figurent parmi les moins rémunérés où le multi emploi et l'expatriation sont parmi les règles de subsistance. Au Vietnam, le secteur de l'éducation, comparé aux autres secteurs, reste peu attractif en raison du faible niveau des salaires. Les jeunes qui sortent de l'université reçoivent une moyenne de trois millions de dongs (114 euros) mensuels, les salaires de la profession étant basés sur l'ancienneté (Mission Étrangère de Paris, 2019). La faible rémunération des enseignant-es rend difficile pour les établissements universitaires d'entrer en compétition avec

\footnotetext{
${ }^{7}$ Source : selon des données sur les comptes nationaux de la Banque mondiale et fichiers de données sur les comptes nationaux de I'OCDE. https://donnees . banquemondiale.org/indicator/NY.GDP.MKTP.CD?locations=VN-KH-LA ${ }^{8}$ D'après la Banque mondiale, sur une moyenne asiatique de $504 \$$ et mondiale de $858 \$$ comme Revenu Mensuel Brut par Habitant en 2016, le RMBH au Cambodge est de 95\$, au Laos de 179\$ et au Vietnam de 172\$.
} 
le secteur privé et de faire face à des universités étrangères financièrement très attractives pour des enseignant-es nationaux/nales hautement qualifié-es.

La qualité de l'enseignement et de la recherche s'en trouve ainsi directement affectée dans la mesure où les connaissances issues de la recherche et les compétences des jeunes diplômées sont mal alignées sur les exigences du marché de l'emploi et la préférence des employeures pour des producteurs/trices de richesse. Même si la demande augmente pour un meilleur accès à l'enseignement supérieur, et que la concurrence pour les enseignant-es qualifié-es des universités s'intensifie, les diplômé-es de certains pays CLV ont encore du mal à trouver un emploi.

Bref, les défis sont multiples et les enjeux sont grands. Alors que des universités tentent de créer de nouvelles dynamiques de recherche par le biais de la coopération internationale, d'autres font appel à des solutions plus orientées vers l'amélioration de la qualité de leur enseignement, à la qualification et à la valorisation professionnelle de leurs cursus pour une meilleure employabilité de leurs diplômé-es. Plusieurs universités, comme celle de Can-The au Vietnam, ont entrepris des études de suivi des diplômé-es et des enquêtes auprès des employeur-es comme moyen d'évaluer la pertinence de leurs programmes et de leurs méthodes d'enseignement. Plusieurs se sont aussi engagées dans des réformes structurelles visant le changement radical de leurs modèles de gouvernance en s'inscrivant dans des programmes de coopération internationale dont nous décrirons quelques-uns dans les sections suivantes.

II va sans dire toutefois que les universités dans les pays CLV gagneront à repenser leurs modèles économiques au niveau des pratiques de rémunération des enseignant-es. Ces universités pourraient s'y prendre en proposant un modèle de rémunération plus attractif pour des professeur-es d'université attiré-es par l'offre privée et l'expatriation. Elles sauraient ainsi réaliser un retour sur investissement en récupérant un temps de travail précieux, de l'énergie créative et de la motivation que des enseignant-es qualifié-es engagent beaucoup plus dans le secteur privé.

II est de rappeler à ce propos que dans plusieurs pays (y compris en France), les enseignantes-chercheurs/euses disposent d'un statut professionnel leur permettant de compléter leurs revenus par des prestations de services rémunérés hors-établissement (cumul de services) sous forme d'expertises, consultations, formations, etc. Or, ce statut est soumis à des règles strictes qui garantissent les intérêts aussi bien de l'enseignant-e (heures supplémentaires et cumul de services) que de l'université (obligation de service et qualité des prestations). Autrement, la dérive au détriment de la qualité des enseignements et de la recherche est vite installée. Un nouveau modèle économique de l'enseignement public qui serait aligné pécuniairement sur le privé ou du moins présentant des avantages de carrière (promotions de grade, responsabilités administrative) est alors très recommandé pour mieux gérer cet aspect de rétributions dans l'enseignement et de la recherche. Plusieurs textes de lois (décrets et 
circulaires) dans les pays CLV sont aujourd'hui à l'étude. Le processus est long mais l'intérêt vaut l'attente car c'est un point crucial et décisif pour réussir les projets de réforme universitaire en cours.

\section{4. ÉTAT DE LA COOPÉRATION INTERNATIONALE}

Les pays de l'ASEAN entretiennent des relations historiques avec les anciennes puissances coloniales de l'Union européenne et des alliances contemporaines avec des États plus au Nord et au Sud du Pacifique qui ont mis en place des systèmes d'enseignement supérieur dans la région, et restent aujourd'hui des partenaires de recherche de premier plan. Pour certaines régions du Sud-Est asiatique, il est évident que de nombreuses réalisations récentes en matière de recherche dépendent de ces liens internationaux (Adam, et al., 2019, p. 7). Une nouvelle stratégie de coopération a été toutefois réinitialisée depuis 1997, lors du $2^{\text {ème }}$ Sommet informel de l'ASEAN à Kuala Lumpur qui a adopté la «Vision 2020 de l'ASEAN »". Par cette vision, les pays concernés avaient opté pour continuer de renforcer leurs projets de coopération pour 2008-2015 et 2016-2025 en mettant fortement l'accent sur les mesures de promotion de la coopération scientifique et technologique pour combler le fossé dans la région (CARI, 2017).

Sous les auspices de cette vision, les liens internationaux de la recherche sont devenus riches et complexes tels les liens du Royaume-Uni avec la Malaisie, des Pays-Bas avec l'Indonésie et notamment de la France avec le Cambodge, le Laos et le Vietnam. La croissance de cette collaboration est motivée en partie par le nombre de programmes internationaux d'aide au développement et de grands projets de financement de la recherche notamment dans les domaines de la santé, de l'environnement et des ressources naturelles, de l'agriculture et de l'éducation, mais aussi par les enjeux culturels et économiques qui accordent à la région un fort potentiels géostratégiques d'avenir. Eu égard à son rôle historique et géostratégique dans la région, la Francophonie prend rapidement position dans ce nouvel échiquier stratégique à travers des projets de la Direction Régionale de l'Aise Pacifique de l'Agence universitaire de la Francophonie (AUF/DRAP).

\section{PROJETS PILOTES DE COOPÉRATION FRANCOPHONE AVEC LES PAYS CLV}

En tant qu'agence universitaire d'envergure internationale, l'AUF fait de l'éducation par le numérique (le numérique éducatif) un socle d'innovation prioritaire dans sa stratégie de coopération internationale. C'est aussi l'axe pivot sur lequel elle fonde ses projets de coopération en Asie du Sud-Est en vue d'appuyer des systèmes éducatifs ayant des difficultés structurelles et des problèmes de gouvernance. Des collaborations étroites en matière de recherche et de formation sont mises en place entre universités francophones du Sud et du Nord avec le soutien d'ONG et d'organisations internationales. Nous introduirons ci-après

\footnotetext{
${ }^{9}$ Déclaration « Asean Vision », https://asean.org/?static_post=asean-vision-2020
} 
quelques-uns des projets pilotes auxquels nous avons contribués en tant qu'expert francophone et partenaire académique d'une collaboration interuniversitaire.

\subsection{L'assurance qualité pour relever le défi de l'éducation et de la recherche}

L'assurance qualité permet à l'institution d'identifier des risques prévisibles, des difficultés et des obstacles pensés comme des menaces au renforcement de la qualité de ses activités, de les analyser et de mettre en place des mesures correctrices et innovantes dans un processus d'amélioration continue. Cette modalité managériale est devenue un leitmotiv dans tous les projets universitaires du Sud-Est asiatique en collaboration avec la DRAP. Thị Cúc Phương Nguyễn, Vice-rectrice de l'Université de Hanoï en donne l'origine. Elle précise qu'un département d'assurance qualité a été créé au sein du Ministère de l'Éducation et de la formation du Vietnam depuis 2003 en vue de produire plusieurs textes réglementaires notamment en matière de référentiels de qualité pour accréditer jusqu'à 2020 des établissements d'enseignement supérieur et des programmes de formation universitaire (Nguyen Thi Cuc, 2017). Les institutions en quête d'accréditation peuvent choisir aussi bien les référentiels vietnamiens (de l'ASEAN) que les standards européens (du HCERES). En 2019, I'Université internationale de Hô Chi Minh-Ville (VNU-HCM) a annoncé qu'elle était devenue la troisième université vietnamienne à satisfaire une évaluation de la qualité universitaire au niveau institutionnel dans le cadre du Réseau d'universités de l'ASEAN (AUN-QA) ${ }^{10}$.

Le soutien du réseau francophone et de l'AUF vient proposer des guides de démarche qualité dans plusieurs domaines : plan de développement stratégique, offre de formation en licence et master et formation doctorale, structuration de la recherche, formation hybride et à distance, prospective universitaire, etc. Ces guides visent une amélioration continue de la qualité et de l'employabilité post-universitaire ainsi que l'alignement sur les niveaux régional et international de la gouvernance universitaire.

L'un des premiers guides d'assurance qualité proposé concerne la structuration de la recherche scientifique en tenant compte de deux processus caractérisant la dynamique de la recherche dans la région à savoir des perspectives d'autonomisation des établissements de recherche, et une volonté de reconnaissance académique et scientifique tant nationale, régionale qu'internationale des projets qu'ils développent. Ce guide pratique, pensé comme un outil à vocation opérationnelle, présente justement les principales orientations nécessaires à la définition et au pilotage d'une démarche-qualité appliquée au service de la structuration de la recherche au sein d'une institution membre de la CONFRASIE. II est doublé d'un guide d'assurance qualité des plans stratégiques de développement d'une institution, ce qui suppose, pour l'institution, de tenir compte de la position qu'elle occupe ou souhaite occuper

\footnotetext{
10 Cf. Le Courrier du Vietnam du 15/02/201, "La qualité des universités vietnamiennes s'améliore ». https://www.lecourrier.vn/la-qualite-des-universites-vietnamiennes-sameliore/599799.html
} 
dans son environnement qu'il soit académique, technologique, concurrentiel, culturel, social, juridique ou économique, qu'il soit local, national, régional ou international.

\subsection{L'innovation pédagogique par la FOAD et la formation hybride}

L'assurance qualité touche aussi à l'enseignement. Dans le cadre de sa mission « numérique éducatif et services à la communauté universitaire francophone », la DRAP a également proposé à ses partenaires universitaires un « Guide d'accompagnement d'une formation hybride » à partir de formations présentielles existantes de niveau Licence, Master ou Doctorat. Ce guide, conçu par l'auteur de cet article, vient comme réponse à une préoccupation majeure de l'AUF de vouloir installer de manière pérenne auprès des établissements universitaires les compétences requises pour concevoir, mettre en œuvre et gérer des formations ouvertes et à distance. II correspond à un processus complet d'opérationnalisation d'une formation hybride, depuis la définition d'une stratégie de mise en œuvre jusqu'à l'évaluation des résultats.

L'objectif principal de ce guide est de permettre aux institutions, qui envisagent une migration vers l'enseignement hybride ou à distance, de définir une démarche qualité dans la définition des objectifs, des résultats attendus et mesurables, des ressources nécessaires pour atteindre des résultats tangibles, des mécanismes d'évaluation et de valorisation des résultats, etc. Ceci suppose la définition des grands axes stratégiques de l'offre de formation hybride et leur intégration dans le modèle de gouvernance de l'établissement (plan de développement stratégique/plan d'action), notamment par la définition d'un socle légal et réglementaire, d'un modèle économique approprié, par la mise en place d'un dispositif de pilotage et de suivi, de référentiels d'accompagnement et de valorisation des acquis d'expérience. Ce guide constitue pour les porteurs de projets de formation par les TIC un document d'orientation sur les choix pédagogiques, technologiques et méthodologiques pour le montage, le suivi et l'évaluation des formations hybrides ou à distance.

\subsection{La prospective universitaire}

Dans la même ligne de préoccupation pour la qualité, la prospective stratégique a été un autre sujet porteur de la coopération AUF/DRAP avec ses partenaires universitaires du Sud-Est asiatique. Un « Guide de prospective stratégique » appliqué à la gouvernance universitaire a été proposé comme cadre régulateur d'un processus défini et mis en œuvre par un établissement d'enseignement supérieur et de recherche pour explorer les futurs possibles et mieux se préparer à l'action de façon responsable.

Ce guide vise un double objectif : d'une part, sensibiliser les institutions membres de la CONFRASIE sur les enjeux liés à la prospective stratégique appliquée à la gouvernance universitaire et, d'autre part, leur permettre d'acquérir les compétences nécessaires à la mise en œuvre d'une démarche prospective. 
Dans le cadre de la construction d'une vision prospective, cette démarche doit permettre à terme de développer une attitude d'anticipation au sein de l'institution et de ses composantes concernées. Elle doit être méthodique, dynamique, inclusive, participative et faire l'objet d'un pilotage responsable de la part de l'équipe de direction de l'institution. Ce qui suppose préalablement d'acquérir la maîtrise des différentes étapes nécessaires à la mise en place de la démarche prospective.

\subsection{La gouvernance universitaire : Projet Erasmus+ PURSEA}

Le dernier projet de coopération francophone en cours (2020-2023) est un projet européen Erasmus+ sur le "Pilotage universitaire rénové dans le sud-est asiatique/PURSEA ». Financé à hauteur d'un million d'euros par la Commission européenne, il se destine, comme énoncé dans son document de cadrage, à permettre à chaque université partenaire de définir et de mettre en œuvre un modèle de gouvernance rénové lui permettant de prendre conscience et d'identifier les mutations et ruptures qui touchent son écosystème, et donc ses propres missions et activités.

Dans une logique de pérennité du modèle à mettre en place, il est prévu en effet de construire une planification stratégique et des plans d'action opérationnels sur une période à définir. ॥ s'agit non seulement de concevoir un modèle de gouvernance universitaire ouvert, pensé comme un dispositif d'aide à la décision, centré sur un pilotage responsable, mais aussi d'inscrire dans cette approche une volonté politique d'autonomisation des universités.

En décidant de relever les défis liés à la définition et la mise en place d'une gouvernance universitaire rénovée et contextualisée, les universités membres du projet PURSEA s'engagent résolument dans une stratégie partenariale et opérationnelle, en tenant compte de la diversité des contextes propres à chacune des quinze institutions partenaires vietnamiennes et cambodgiennes en s'appuyant sur la richesse de l'expertise des sept universités partenaires européennes : CNRS de Grenoble, Université de Lorraine, Université de Bretagne Occidentale, Université de Toulon, Université de Bordeaux Montaigne, Université Libre de Bruxelles et Université de Duisburg-Essen.

À travers l'appui du programme ERASMUS+, le projet PURSEA se donne pour ambition de mutualiser les savoirs et savoir-faire en matière de gouvernance universitaire rénovée et contextualisée afin de relever des défis depuis la détermination du plan de développement stratégique jusqu'à la mise en place des plans d'action opérationnels, en co-construisant des méthodologies rigoureuses accompagnées d'outils pertinents.

L'appui du programme ERASMUS+ au renforcement des capacités en enseignement supérieur devient en effet nécessaire pour repenser à plusieurs et en profondeur les orientations stratégiques des plans de développement des établissements d'enseignement supérieur en fonction du degré d'autonomisation, concept multidimensionnel actuellement mis en place dans les deux pays partenaires du projet, le Vietnam et le Cambodge, à savoir 
autonomie dans la définition d'un nouveau modèle économique, autonomie dans la définition d'une gestion prospective des ressources humaines, autonomie dans la définition d'une politique partenariale, etc. Ceci explique le caractère doublement structurant du projet tant par son ambition que par son ampleur. Par son ambition, l'appui du programme ERASMUS+ crée les conditions d'une transformation en profondeur d'une gouvernance universitaire rénovée en relation avec l'environnement propre à chaque université ; par son ampleur, il permet la structuration et le développement d'une expertise qui vise l'élaboration et la mise en place de livrables au service du plan de développement stratégique des universités.

Au-delà de la durée du projet, il s'agit de créer les conditions en vue de permettre aux deux systèmes universitaires cambodgien et vietnamien, et aux universités qui y sont rattachés, de bénéficier d'une offre d'expertise à partir des livrables produits au cours du projet.

\section{CONCLUSION}

II serait difficile de fournir un résumé simple et complet de l'état actuel de l'environnement de l'éducation et de la recherche dans les pays du Sud-Est asiatique, tellement le contexte est polymorphe et composite. Les systèmes de l'enseignement supérieur dans les pays CLV font aujourd'hui face à plusieurs défis quantitatifs et qualitatifs (Cerbelle, 2016). Mais d'aucun ne peut nier que durant les deux dernières décennies, la réforme de l'enseignement supérieur fût une priorité pour la plupart de ces pays en réponse à leur rapide développement économique et aux nouvelles modalités de leurs échanges commerciaux. Le Vietnam a fixé pour cela des affinités très ambitieuses pour répondre à la demande du marché de l'emploi et concurrencer les pays voisins de l'ASEAN. II a promulgué des textes de loi et mis en place une stratégie qui tend vers un modèle " standard » inscrit dans un mouvement d'ensemble tout en poursuivant des orientations similaires aux réformes européennes même si ce mouvement s'inscrit dans un contexte particulier, différent du contexte dans lequel se sont développées les réformes en Europe.

Dans sa globalité, la situation des universités des pays CLV présente une image complexe avec des contrastes très marqués. Certains pays ont connu une croissance économique très rapide (sans considérer la période de crise du Covid-19) comme le Vietnam $\left(4,8 \%{ }^{11}\right.$ ) et le Laos $(6,5 \%)$. En revanche, les activités et les résultats de la recherche restent faibles malgré l'exception vietnamienne mentionnée précédemment. La possibilité d'un réseau régional de collaboration inter-universitaire réellement efficace n'a pas encore été pleinement exploitée. Certains pays ne disposent tout simplement pas de capacités suffisantes pour s'engager même au niveau local en vue de contenir les différences régionales, et entre zones rurales et urbaines. Pourtant, les niveaux de collaboration sont importants pour la majeure partie de la région, notamment la mise en œuvre depuis novembre 1995 de l'Asean University Network

\footnotetext{
${ }^{11}$ Cf. Le Courrier du Vietnam du 04/04/2020. http://www.lecourrier.vn/la-croissance-economique-du-vietnamprevue-a-48-en-2020/725505.html
} 
(AUN) avec un « programme pour harmoniser l'enseignement supérieur en Asie du Sud-Est » (Yonezawa, et al., 2014, p. 92). II reste encore beaucoup à faire pour traduire cela dans une meilleure croissance des domaines de la recherche et de l'enseignement supérieur.

Trois apports essentiels permettraient néanmoins de combler l'écart de performance et de développement dans le cadre de partenariats non seulement régionaux, mais aussi des liens avec le reste du monde :

- D'abord, mieux investir dans le capital humain d'autant que le nombre relatif de chercheurs/euses sur le marché du travail est encore très faible et doit être lié à une meilleure stratégie de financement et de régulation juridique de l'enseignement supérieur ;

- Ensuite, promouvoir un environnement de recherche fortement encouragé par le biais des universités libres pour qu'elles poursuivent leurs propres programmes d'innovation scientifique et technologique ;

- II faudrait surtout suivre le rythme de la prospérité des autres pays riches de la région en développant une économie de la connaissance. Les pays CLV ne peuvent pas se permettre de sous-investir dans l'enseignement et la recherche scientifiques et technologiques surtout qu'ils pourraient mobiliser d'énormes ressources humaines et financières avec un potentiel de rénovation considérable.

II est essentiel que tout cela permette non seulement de mener des recherches de pointe, mais également de créer une transformation éducative et sociale plus solide grâce aux capacités en ressources humaines. L'ampleur de ce changement et sa traduction dans différents domaines de recherche constitueront un défi majeur à relever :

Les plans et les budgets doivent s'aligner de manière à soutenir à la fois la mise en œuvre efficace et le suivi de la réforme de l'éducation. Toutes les politiques et tous les programmes éducatifs doivent être coordonnés au sein du secteur de l'éducation et avec d'autres ministères concernés tels que ceux s'occupant du développement économique, du développement des ressources humaines, du travail, des sciences et technologies, de l'agriculture, etc. (UNESCO, 2014, p. 69)

\section{RÉFÉRENCES}

Adam, J., Pendlebury, D., Rogers, G., \& Szomszor, M. (2019). Global Research Report - South and Southeast Asia (rapport n WS385512528/01). Philadelphia: ISI, Institute for Scientific Information. https://clarivate.com/webofsciencegroup/download/39371/

Banbang, I. (2017). An analysis of the Asean cooperation in science and technology. AEC Blueprint 2025 Analysis, 1(25), 2-14.

Ben Henda, M. (2016a). Formation à distance et outils numériques pour l'enseignement supérieur et la recherche en Asie-Pacifique (Cambodge, Laos, Vietnam). Partie 01 : État des lieux. Hanoï: Bureau Asie pacifique de l'Agence universitaire de la Francophonie. https://hal.archives-ouvertes.fr/hal-01475554 
Ben Henda, M. (2016b). Identification des besoins en formation tic/e dans les pays francophones du sud. Étude réalisée par: Initiatives pour le Développement numérique de l'espace universitaire francophone. Paris: Agence universitaire de la Francophonie. https://hal.archives-ouvertes.fr/hal-01475539

Carroué, L. (2015). L'éducation en Asie aux défis du développement. Revue internationale d'éducation de Sèvres, 68, 61-68. https://doi:10.4000/ries.4343

Cerbelle, S. (2016). L'enseignement supérieur au Vietnam : les enjeux d'une réforme inspirée des orientations occidentales. Cahiers de la recherche sur l'éducation et les savoirs, 15, 239-262. http://journals.openedition.org/cres/2968

David, M. E., \& Amey, M. J. (2020). The SAGE Encyclopedia of Higher Education. London: SAGE Publications Ltd.

Hirosato, Y., \& Kitamura, Y. (Ed.). (2009). The Political Economy of Educational Reforms and Capacity Development in Southeast Asia: Cases of Cambodia, Laos and Vietnam. Dordrecht: Springer Science \& Business Media.

Ministère de l'Europe et des Affaires Étrangères, France. (2018). Fiche Vietnam. Hanoï : Ambassade de France au Vietnam.

https://www.diplomatie.gouv.fr/IMG/pdf/2018.11_fiche_curie_vietnam_cle03c4c8.pdf

Mission Étrangère de Paris. (2019). La lutte des jeunes enseignants face à la jungle de l'éducation vietnamienne. Hanoï : Église d'Asie, Mission étrangère de Paris. https://missionsetrangeres.com/eglises-asie/la-lutte-des-jeunes-enseignants-face-a-lajungle-de-leducation-vietnamienne/

Nguyen Tan, D. (2017). Les TIC au service de la qualité des formations: Le cas des programmes vietnamiens évalués par l'ASEAN University Network. Thèse de doctorat, Université de Strasbourg. https://tel.archives-ouvertes.fr/tel-01599708

Nguyen Thi Cuc, P. (2017, 30 novembre). Les défis de l'enseignement supérieur au Vietnam. Innovation pédagogique.

https://theconversation.com/les-defis-de-lenseignement-superieur-au-vietnam-88283

Nguyen Thi Yhanh, H. (2015). Mesurer la performance des universités au Vietnam en termes d'efficience: Une application de la méthode DEA. Thèse de doctorat, Université de Rennes 1.

https://ecm.univ-rennes1.fr/nuxeo/site/esupversions/349200d0-317a-45ff-8242f24fc04b65da

Nguyen, T. V., Ho-Le, T. P., \& Le, U. V. (2017). International Collaboration in Scientific Research in Vietnam: An Analysis of Patterns and Impact. Scientometrics, 110(2), 1035-1051. https://doi.org/10.1007/s11192-016-2201-1

UNESCO, \& UNESCO Bangkok Office. (2014). Education Systems in ASEAN+6 Countries: A Comparative Analysis of Selected Educational Issues. Paris: UNESCO. https://www.right-to-education.org/sites/right-to-education.org/files/resourceattachments/UNESCO_Education_Systems_in_Asia_Comparative_Analysis_2014.pdf

Vuong, Q.-H. (2019). The Harsh World of Publishing in Emerging Regions and Implications for Editors and Publishers: The Case of Vietnam. Learned Publishing, 32(4), 314-324. https://doi.org/10.1002/leap.1255 
Yonezawa, A., Kitamura, Y., Meerman, A., \& Kuroda, K. (2014). Emerging International Dimensions in East Asian Higher Education. Dordrecht: Springer Science \& Business Media.

Open Access Publications - Bibliothèque de l'Université de Genève Creative Commons Licence 4.0

(c) (1) 